\title{
The Effects of Time Framing on Compliance to Hypothetical Social-Distancing Policies Related to COVID-19
}

\author{
Michael J. Harman ${ }^{1}$
}

Accepted: 29 October 2020 / Published online: 3 January 2021

(C) Association for Behavior Analysis International 2021

\begin{abstract}
The current study analyzed the effects of two frames for durations of time - calendar unit and calendar date - on measures of compliance to hypothetical social-distancing policies related to the COVID-19 pandemic. Participants reported the extent to which they would comply with hypothetical social-distancing policies lasting different durations of time. Durations of time were framed as calendar units (e.g., days, weeks, months, years) and calendar dates (i.e., specific dates the policies would extent to). Levels of compliance across durations of time were used to calculate the area under the curve $(\mathrm{AuC})$ for each condition. Social-distancing policies framed in calendar dates yielded significantly greater AuC values compared to social-distancing policies framed in calendar units. Participants' self-reported political affiliation yielded a significant main effect: Conservative participants' AuC values were significantly lower than liberal participants' AuC values. The framing of the duration of time was a significant variable in controlling rates of compliance to hypothetical social-distancing policies.
\end{abstract}

Keywords Compliance $\cdot$ Delay discounting $\cdot$ Framing $\cdot$ Social policy $\cdot$ Treatment integrity

Delay discounting refers to the decrease in the perceived value of a reward because of a delay in accessing the reward (McKerchar \& Renda, 2012). That is, when presented with a small-magnitude reward and a large-magnitude reward, an individual may choose the small-magnitude reward if access to that reward is more immediate than access to the large-magnitude reward. The hypothesized behavioral mechanism for this choice is that the delay to consuming or accessing the reward reduces the subjective value of the larger, delayed consequence in comparison to the smaller, immediate consequence. In general, a choice that results in a small but immediate reward at the cost of a delayed but larger reward is termed impulsive, whereas a choice that results in

Michael J. Harman

Michael.Harman@briarcliff.edu

1 Department of Psychology, Briar Cliff University, Sioux City, IA 51104, USA 
a delayed but larger reward at the cost of an immediate but small reward is termed selfcontrol (Madden \& Johnson, 2010).

Similar behavioral mechanisms may also help to explain why individuals engage in potentially risky behavior in the context of immediate and delayed rewards (i.e., probability and delay discounting; Mazur, 1985; Mishra \& Lalumière, 2017). For example, an individual may choose an immediately available reinforcer (e.g., unprotected sex; Collado, Johnson, Loya, Johnson, \& Yi, 2017) even if it is associated with a highly probable aversive event (e.g., sexually transmitted infection). In general, a choice that results in an immediate reward but a high probability of an aversive event is termed risk prone, whereas a choice that results in a delayed reward but a low probability of an aversive event is termed risk averse.

Discounting is typically studied within the context of hypothetical scenarios with money (e.g., Monetary Choice Questionnaire; Kirby \& Marakovic, 1996); however, research in the explanatory and predictive utility of discounting mechanisms has recently been used to understand phenomena such as support for hypothetical public policy decisions (e.g., Hardisty, Johnson, \& Weber, 2010; Hardisty \& Weber, 2009; Plumm, Borhart, \& Weatherly, 2012). For example, Plumm et al. (2012) presented participants with several delayed hypothetical legal resolutions to culturally relevant social-policy issues (e.g., bills for the legalization of gay marriage, affirmative action, abortion, doctor-assisted suicide). Participants were instructed to self-report the minimum percentage of the resolution that they would be willing to accept for the bill to pass immediately instead of waiting for the bill to pass in its entirety. In general, participants' self-reported percentages followed a typical discounting curve whereby relatively short delays yielded higher percentages (i.e., more willing to wait) and relatively long delays yielded lower percentages (i.e., less willing to wait).

Researchers have begun to apply discounting frameworks in a variety of clinical contexts beyond hypothetical scenarios to understand pathologies such as drug abuse (e.g., Bickel \& Marsch, 2001; Kirby, Petry, \& Bickel, 1999), obesity (e.g., Epstein, Salvy, Carr, Dearing, \& Bickel, 2010), gambling (e.g., Petry \& Madden, 2010), and impulsive disorders (see Jackson \& MacKillop, 2016, for a review). The general findings from these studies indicate that impulsive tendencies may underlie pathological behavioral patterns (see Bickel, Jarmolowicz, Mueller, Koffarnus, \& Gatchalian, 2012, for review). Accordingly, a growing body of research within the discounting literature has begun to focus on interventions to reduce impulsive decision making (see Rung \& Madden, 2018, for a review and meta-analysis). One intervention that is gaining empirical evidence concerns framing. Framing refers to interventions that alter the way a scenario or outcome is perceived (see Koffarnus et al., 2013, for a review). Framing manipulations often result in different discounting patterns despite no actual change to the parameters of the scenario (e.g., DeHart \& Odum, 2015; LeBouf, 2006; Read, Frederick, Orsel, \& Rahman, 2005). For example, DeHart and Odum (2015) compared the effects of the framing of delays as specific dates in the future (e.g., March 23) to the framing of delays in standard calendar units (e.g., in 6 months) on the rate at which participants discounted hypothetical monetary rewards. Time framed as specific dates resulted in less discounting compared to the calendar units. 
The social significance of reducing impulsive decision making is especially relevant when impulsive behaviors pose significant risks to public health (Rung $\&$ Madden, 2018). For example, citizens across the world are now contacting novel social policies because of the COVID-19 pandemic. Among these policies are regulations for social distancing (e.g., stay-at-home orders). These policiesenacted at national, state, and local levels-generally enforce or encourage limits on access to social contact for extended intervals of time. The function of these policies is to reduce the spread of the SARS-CoV-2 virus by avoiding or limiting as much social contact as possible (Centers for Disease Control and Prevention [CDC], 2020; World Health Organization [WHO], 2020).

Similar to other behavior-analytic interventions, the efficacy of social-distancing policies is limited to the extent to which individuals implement the policy with high treatment integrity. In the behavior-analytic literature, an intervention is generally considered to have high treatment integrity and remain efficacious if at least $80 \%$ of the treatment components are implemented correctly (e.g., Bottini, Morton, Gillis, \& Romanczyk, 2020; Hirst \& DiGennaro Reed, 2015; St. Peter Pipkin, Vollmer, \& Sloman, 2010). In the context of social distancing, the CDC has outlined several components necessary for implementing social distancing with high integrity (Centers for Disease Control and Prevention, 2020): (a) Limit social interaction to individuals within a household, (b) maintain a distance of at least $6 \mathrm{ft}(\sim 2 \mathrm{~m})$ between individuals, (c) only leave the household for essential activities, and (d) when in public, wear a face mask. Although a number of factors may contribute to deficits in treatment integrity (e.g., response effort, training methods), the duration of time for which individuals are encouraged to practice social distancing is especially relevant when considering the behavioral mechanisms responsible for sustained compliance over increasing durations of time. That is, implementing social distancing with high integrity requires that individuals consistently forgo typical sources of immediate social reinforcement (e.g., social gatherings) and instead emit behaviors controlled by delayed sources of reinforcement (e.g., lower infection rates). However, impulsive decision making may limit the efficacy of policies designed to reduce the severity of the COVID-19 pandemic. For example, despite the severity of the pandemic and the encouragement to practice social distancing, individuals may continue to choose to attend social gatherings despite a high probability of exposure to a dangerous infectious disease. From a discounting perspective, the subjective value of immediate social reinforcement (e.g., gathering with friends) is greater than the high probability of delayed aversive consequences (e.g., illness because of exposure to infectious disease). Given that impulsive decision making may limit the efficacy of socialdistancing policies, interventions that alter choice allocation in typical discounting scenarios may alter compliance with social-distancing policies requiring high integrity across increasing durations of time.

The current study investigated the effects of time framing on compliance with hypothetical social-distancing policies similar to those enacted during the COVID19 pandemic. Specifically, this experiment examined the effect of framing durations of hypothetical social-distancing policies as calendar units or calendar dates on participants' self-reported rates of compliance with policies. Rates of compliance were transformed into measures of treatment integrity based on an $80 \%$ compliance criterion. 


\section{Method}

\section{Participants}

One hundred participants were recruited from the pool of workers organized by Amazon's Mechanical Turk (Behrend, Sharek, Meade, \& Wiebe, 2011). All participants were 18 years of age or older and held an IP address in the United States. Participants were compensated $\$ 0.20$ for completing the survey within the allotted time ( 5 min.). After the data omission criteria were applied (see below), the final sample consisted of 92 participants. See Table 1 for relevant demographic information reported by the participants.

\section{Materials}

Two questionnaires were used to measure self-reported levels of compliance with hypothetical social-distancing policies. Questionnaires were developed in Qualtrics (Qualtrics, 2020, Provo, Utah). Both questionnaires included the following introduction:

Table 1 Participant Demographics and Descriptive Statistics

\begin{tabular}{|c|c|c|c|c|}
\hline & $N$ & Mean AuC: Unit & Mean AuC: Date & Mean AuC: Money \\
\hline \multicolumn{5}{|l|}{ Gender } \\
\hline Male & 45 & $0.40(0.40)$ & $0.54(0.38)$ & $0.68(0.27)$ \\
\hline Female & 42 & $0.50(0.38)$ & $0.58(0.39)$ & $0.65(0.22)$ \\
\hline Not specified & 5 & $0.85(0.27)$ & $0.97(0.06)$ & $0.68(0.19)$ \\
\hline \multicolumn{5}{|l|}{ Age range } \\
\hline $18-25$ & 11 & $0.32(0.44)$ & $0.50(0.43)$ & $0.57(0.30)$ \\
\hline $26-35$ & 43 & $0.44(0.38)$ & $0.52(0.36)$ & $0.68(0.24)$ \\
\hline $36-54$ & 28 & $0.50(0.39)$ & $0.61(0.41)$ & $0.70(0.24)$ \\
\hline $55+$ & 10 & $0.67(0.37)$ & $0.90(0.19)$ & $0.61(0.18)$ \\
\hline \multicolumn{5}{|l|}{ Political affiliation } \\
\hline Conservative & 26 & $0.24(0.27)$ & $0.39(0.32)$ & $0.71(0.24)$ \\
\hline Liberal & 57 & $0.58(0.39)$ & $0.70(0.36)$ & $0.65(0.24)$ \\
\hline Not specified & 9 & $0.44(0.44)$ & $0.46(0.42)$ & $0.68(0.26)$ \\
\hline \multicolumn{5}{|l|}{ Reported risk } \\
\hline Extremely likely & 9 & $0.50(0.40)$ & $0.59(0.35)$ & $0.69(0.30)$ \\
\hline Moderately likely & 14 & $0.51(0.41)$ & $0.59(0.40)$ & $0.67(0.23)$ \\
\hline Slightly likely & 25 & $0.50(0.40)$ & $0.68(0.37)$ & $0.71(0.21)$ \\
\hline Neutral & 13 & $0.44(0.41)$ & $0.43(0.41)$ & $0.71(0.16)$ \\
\hline Slightly unlikely & 15 & $0.40(0.40)$ & $0.55(0.39)$ & $0.63(0.28)$ \\
\hline Mod. unlikely & 10 & $0.49(0.42)$ & $0.64(0.41)$ & $0.54(0.31)$ \\
\hline Extremely unlikely & 6 & $0.37(0.38)$ & $0.50(0.40)$ & $0.68(0.17)$ \\
\hline
\end{tabular}

Note. Sample distributions are detailed for each between-subjects variable. Standard deviations for measures of central tendency are in the adjacent parentheses. $\mathrm{AuC}=$ area under the curve. 
Introduction: Imagine that you are a citizen living in a country that is currently responding to a worldwide infectious disease pandemic. At the moment, there is no cure/vaccine for the disease. However, medical experts suggest that minimizing social contact can significantly reduce your chance of contracting the disease. Furthermore, by minimizing social contact, fewer people, at any given time, may require immediate emergency help. Thus, minimal social contact will decrease your likelihood of contracting the disease and will increase the ability of medical professionals to effectively treat individuals who have contracted the disease.

Scenario: A team of internationally renowned medical doctors state that the severity of the pandemic will be significantly reduced to manageable levels (i.e., similar to the management of the seasonal influenza virus) if citizens comply with social-distancing policies (confine themselves to contact just with individuals in their household) for a certain duration of time. For example, individuals are encouraged to avoid crowds, traveling, and all other contexts in which 10 or more people are present.

For each of the following scenarios, assume that the team of medical experts has determined the optimal duration of time to engage in social distancing. That is, the team predicts that if people comply with the proposed duration, rates of infections will be reduced to manageable levels. For each duration, please report how likely you are to comply with the proposed social-distancing suggestion. You will report your likelihood of complying on a scale of 0 (not comply at all) to 100 (comply completely for the full duration).

Important: For the following scenarios, please imagine that today's date is March 15, 2020.

After participants responded to the first two questions, a screen prompted participants to "remember to imagine that today's date is March 15, 2020." After participants responded to six questions, participants were required to respond to a "pop quiz" question that instructed them to select the date they were previously instructed to imagine as today's date. Responses to the pop quiz ensured that participants were correctly interpreting the durations of time in the calendar date condition. Participants selected from one of three dates. No feedback was provided for correct or incorrect selections.

\section{Calendar Unit Condition}

Participants completed an eight-item questionnaire that was used to measure their selfreported levels of compliance with hypothetical social-distancing policies. Each question prompted participants to select the likelihood that they would comply with a hypothetical social-distancing policy. The phrasing of each question is similar to that used in Plumm et al. (2012). Across questions, the policies differed in the duration of time that the policy would be in effect. Eight durations were used: 1 day, 1 week, 2 weeks, 1 month, 3 months, 6 months, 9 months, and 1 year. The durations were framed according to the relevant calendar unit and were presented in a random order for each participant (see Appendix A). Participants responded to each question by adjusting a slider on a scale from 0 (not comply at all) to 100 (fully comply). Tick marks and values 
were present every five units. Adjusting the slider position resulted in a synchronous change in value above the slider. For each question, the slider started in the 0 position.

\section{Calendar Date Condition}

Participants completed a questionnaire that was identical to the questionnaire in the calendar unit condition with one exception. The durations of the hypothetical socialdistancing policies were framed according to the calendar date that the policy would extend to (see Appendix B). Eight dates were used: March 16, 2020 (1 day); March 22, 2020 (1 week); March 29 (2 weeks); April 15, 2020 (1 month); June 15, 2020 (3 months); September 15, 2020 (6 months); December 15, 2020 (9 months); and March 15, 2021 (1 year).

\section{Procedure}

After reading information about the study and agreeing to participate, participants responded to demographic questions. Demographic questions collected information on state of residence, age range (18-25, 26-35, 36-54, and 55+ years), gender (male, female, other), political affiliation (liberal, conservative, other), and self-reported risk of contracting COVID-19 (extremely likely, moderately likely, slightly likely, neutral, slightly unlikely, moderately unlikely, extremely unlikely). Participants then completed the two questionnaires in a random order. After completing the first questionnaire and before beginning the second questionnaire, participants completed a brief monetarydiscounting questionnaire (data available from the author at request). The monetarydiscounting questionnaire was identical to that of Plumm et al. (2012) and was included to control for carryover effects between the two social-distancing questionnaires. Following the completion of the three questionnaires, participants were thanked for their time and given a password to arrange for compensation.

\section{Data Analysis}

Participants' self-reported levels of compliance for the calendar unit and calendar date conditions were first transformed based on the $80 \%$ integrity criterion. That is, at each delay, compliance rates were assigned a value of 1 if self-reported compliance was equal to or greater than 80 and a value of 0 if less than 80 . Furthermore, the durations of the hypothetical social-distancing policies were standardized by dividing each duration by 365. Participants' transformed discounting curves for each condition were used to calculate a measure of the area under the curve (AuC; Myerson, Green, \& Warusawitharana, 2001; see equation 1):

$$
A u C=\sum\left(x_{2}-x_{1}\right)\left[\frac{y_{1}+y_{2}}{2}\right]
$$

where $x_{2}$ and $x_{1}$ refer to adjacent standardized durations of social distancing, and $y_{1}$ and $y_{2}$ refer to adjacent transformed self-reported levels of compliance (i.e., 0 or 1 ) at $x_{1}$ and $x_{2}$, respectively. A repeated-measures $t$ test was used to analyze whether significant differences were present between mean AuC values. Subsequent analyses incorporated 
the following variables into a mixed-measures factorial analysis of variance (ANOVA) as between-subjects factors: gender, age, political affiliation, and self-reported risk of contracted COVID-19. Finally, Pearson correlation analyses were conducted to determine the extent to which death rates (according to the CDC at the time of this study) from participants' self-reported state of residence correlated to the $\mathrm{AuC}$ values for compliance with social-distancing policies in both conditions. For the aforementioned inferential statistics, an initial alpha value of $\alpha=0.05$ was used to determine statistical significance. For the four mixed-measures factorial ANOVAs, an adjusted alpha value of $\alpha=0.01$ (Bonferroni correction) was used to control for the increased probability of a Type I error associated with multiple analyses.

A participant's data were not included in the final analyses if (a) the participant did not provide a response during any of the two questionnaires, or (b) the participant provided an incorrect answer to the "pop quiz" question concerning the date. Six participants' data were omitted due to missing responses, and two participants' data were omitted due to incorrect answers to the pop quiz. Thus, data from 92 participants were included in the final analyses.

\section{Results}

The calendar date condition yielded a higher percentage of participants with at least $80 \%$ compliance at each hypothetical social-distancing duration compared to the calendar unit condition (see Fig. 1). Data paths resemble discounting-like curves, whereby changes in the dependent variable (i.e., percentage of participants complying with the policy) decrease nonlinearly with increases in the independent variable (i.e., duration of the policy). The rate at which the dependent variable decreases is dependent on the condition: The calendar date condition yielded a relatively shallow curve, whereas the calendar unit condition yielded a relatively steep curve. The greatest difference between conditions was at the 1-year duration $\left(M_{\text {Date }}=0.46, S D=0.49\right.$; $\left.M_{\text {Unit }}=0.30, S D=0.46\right)$, and the smallest difference between conditions was at the 1month duration $\left(M_{\text {Date }}=0.78, S D=0.41 ; M_{\text {Unit }}=0.74, S D=0.44\right)$.

Figure 2 shows the mean, median, and quartile distributions of $\mathrm{AuC}$ measures for each condition. A repeated-measures $t$ test determined that the mean AuC measure for the calendar date condition $(M=0.58, S D=0.38)$ was significantly higher than the mean AuC measure for the calendar unit condition $(M=0.47, S D=0.39), t(91)=4.20$, $p<0.001, d=0.30$. This difference represents a small to medium effect size (Cohen, 1988).

Four separate mixed-measures factorial ANOVAs were conducted to determine the extent to which between-subjects variables yielded independent main effects or interactions. Table 2 lists the between-subjects variables and the levels each yielded (see Table 1 for descriptive statistics). In each analysis, the frame condition consistently yielded a significant main effect $(p s \leq 0.001)$ with a large effect size $\left(\eta^{2} \geq 0.13\right.$; Cohen, 1988). Political affiliation (conservative or liberal) was the only between-subjects variable to yield a significant main effect, $F(1,81)=17.19, p<0.001, \eta^{2}=0.18$. Figure 3 shows the distribution of $\mathrm{AuC}$ values for each condition for participants selfidentifying as conservative $(N=26)$ or liberal $(N=57)$. The AuC values for both the calendar date condition and the calendar unit condition were greater for participants 


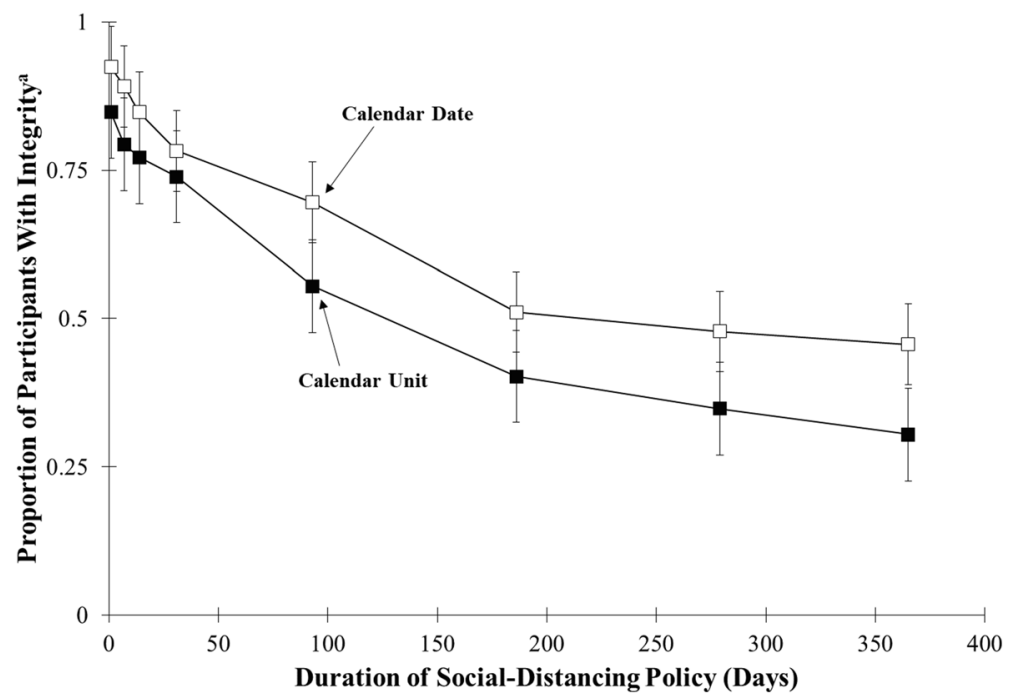

Fig. 1 Proportion of Participants Reporting Integrity Across Durations of Social-Distancing Policies. Note. Proportions were based on the distribution of participants scoring a 1, per the transformation described in the Data Analysis section, at each duration. Error bars correspond to standard deviations. ${ }^{\text {a }}$ Integrity was based on self-reported compliance at or above $80 \%$

self-identifying as liberal $\left(M_{\text {Date }}=0.69, S D=0.37 ; M_{\text {Unit }}=0.58, S D=0.39\right)$ compared to conservative $\left(M_{\text {Date }}=0.39, S D=0.33 ; M_{\text {Unit }}=0.24, S D=0.27\right)$. For both political affiliations, however, the calendar date condition yielded significantly greater mean AuC values - conservative: $t(25)=2.41, p=0.24, d=0.52$; and liberal: $t(56)=3.37, p$ $=0.001, d=0.31$ ).

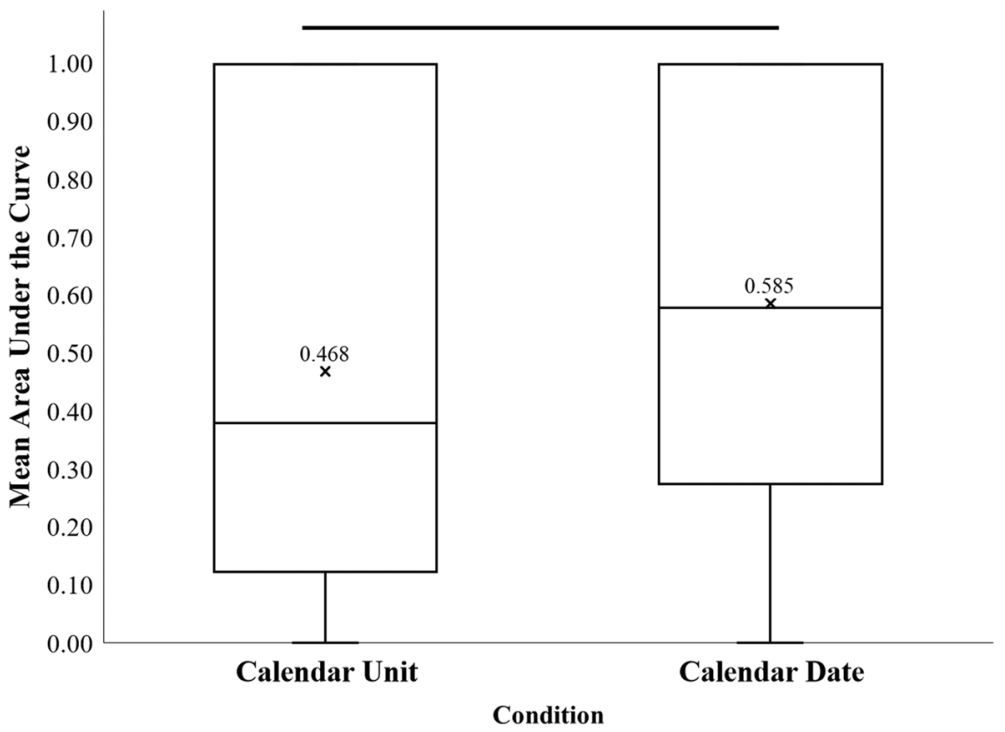

Fig. 2 Distribution of Participants' AuC Measures in the Calendar Unit Condition and the Calendar Date Condition. Note. The crosses indicate the mean area under the curve (AuC) value for each condition. The bold line indicates a significant difference between AuC values $(p<0.001)$ 
As it concerns the correlation between death rates from participants' self-reported states of residence and compliance with social-distancing policies, correlation coefficients were nonsignificant for both conditions $\left(r_{\text {Date }}=0.09, p>0.05 ; r_{\text {Unit }}=0.10, p>\right.$ $0.05)$. Thus, death rates of the states in which participants resided were not correlated to the self-reported levels of compliance with social-distancing policies framed as calendar dates or calendar units.

\section{Discussion}

The results of the current study extend the effects of framing to compliance with hypothetical social-distancing policies. Specifically, this study demonstrated that durations of hypothetical social-distancing policies framed as calendar dates yield greater rates of compliance than durations of social-distancing policies framed in calendar units. This finding replicates previous work on delay framing (DeHart \& Odum, 2015; LeBouf, 2006; Read et al., 2005). Similar to Plumm et al. (2012), participants' selfreported political affiliation exerted a significant main effect on rates of hypothetical compliance. That is, self-identifying conservative participants had lower overall rates of hypothetical compliance compared to self-identifying liberal participants. This finding is difficult to interpret from a behavior-analytic standpoint. That is, these subjectspecific variables are not feasible variables to manipulate in any experimental analysis. Furthermore, previous research has yielded inconsistent conclusions regarding the effects of political affiliation on measures of delay discounting. On the one hand, previous research has found that individuals with self-reported liberal ideologies demonstrate shallower discounting for delayed outcomes (Hardisty et al., 2010; Rattner, Yagil, \& Shermn-Segal, 2003). On the other hand, Weatherly (2010) reported that individuals with self-reported liberal ideologies demonstrated steeper discounting. In light of the current study's findings and the unreliable findings in previous research, future studies should investigate the conditions under which political affiliation affects measures of delay discounting.

Despite being medically significant variables (CDC, 2020; WHO, 2020), participants' self-reported age and level of risk for contracting COVID-19 did not exert any

Table 2 Inferential Statistics From Mixed-Factorial ANOVAs

\begin{tabular}{|c|c|c|c|c|}
\hline BS variable & Levels & Main effect: Frame & Main effect: BS & Interaction \\
\hline $\begin{array}{l}\text { Political } \\
\quad \text { affiliation }\end{array}$ & 2 & $\begin{array}{l}F(1,81)=16.42, p<0.001, \eta^{2}= \\
\quad 0.17\end{array}$ & $\begin{array}{l}F(1,81)=17.19, p<0.001 \\
\quad \eta^{2}=0.18\end{array}$ & $\begin{array}{l}F(1,81)=0.33, p= \\
\quad 0.57\end{array}$ \\
\hline Gender & 2 & $\begin{array}{l}F(1,85)=15.85, p<0.001, \eta^{2}= \\
\quad 0.16\end{array}$ & $F(1,85)=0.82, p=0.37$ & $\begin{array}{l}F(1,85)=1.07, p= \\
\quad 0.31\end{array}$ \\
\hline $\begin{array}{l}\text { Self-reported } \\
\quad \text { risk }\end{array}$ & 7 & $\begin{array}{l}F(1,85)=12.66, p<0.001, \eta^{2}= \\
\quad 0.13\end{array}$ & $F(6,85)=0.38, p=0.89$ & $\begin{array}{l}F(6,85)=0.94, p= \\
\quad 0.47\end{array}$ \\
\hline Age range & 4 & $\begin{array}{l}F(1,88)=20.02, p<0.001, \eta^{2}= \\
\quad 0.19\end{array}$ & $F(3,88)=2.52, p=0.63$ & $\begin{array}{l}F(3,88)=1.05, p= \\
\quad 0.38\end{array}$ \\
\hline
\end{tabular}

Note. Each between-subjects (BS) variable was included in a separate mixed-factorial analysis of variance (ANOVA) with the condition frame as the within-subjects variable. Measures of effect size are included for significant findings. 


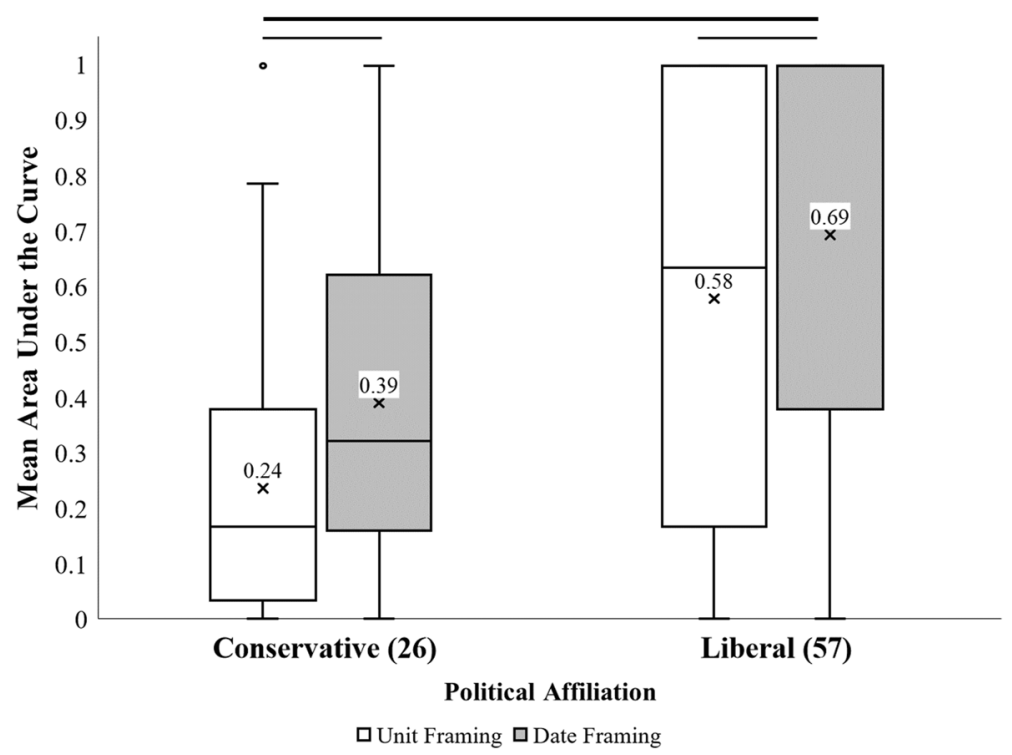

Fig. 3 Distribution of Participants' AuC Measures Based on Self-Reported Political Affiliation. Note. The crosses indicate the mean area under the curve $(\mathrm{AuC})$ values. The bold black line indicates a significant main effect of political affiliation $(p<0.001)$. The less weighted gray lines indicate significant differences between mean AuC values within each group $(p<0.025)$. Numbers in parentheses indicate the sample size for each group

significant effect on rates of hypothetical compliance. There was, however, an increasing trend in $\mathrm{AuC}$ measures across age groups, with participants identifying as 55+ years old having the highest rates of hypothetical compliance and participants identifying as 18-25 years old having the lowest rates of hypothetical compliance. Future research should seek to identify the conditions under which self-reported age and level of risk are and are not significant predictors of rates of compliance with social-distancing policies.

The findings of this study occasion at least three potential explanations. First, it is possible that the calendar date condition yielded shallower discounting curves because the specific calendar dates evoked spontaneous verbal behavior (cf. Diaz \& Berk, 1992) that functioned similarly to episodic future thinking (EFT; Atance \& O'Neil, 2001). EFT involves participants vividly imagining themselves engaging in behavior at a future date while responding to a delay-discounting task (Bromberg, Lobatcheva, \& Peters, 2017; O’Donnell, Hollis-Hansen, \& Epstein, 2019). For example, Bromberg et al. (2017) instructed participants to report personal future events at delays used in a standard hypothetical monetary delay-discounting task. During half of the trials, the delays included a tagline prompting participants to imagine themselves engaging in a personal event at the particular delay. Discounting curves for delayed hypothetical money were significantly shallower when participants were prompted to imagine temporally similar future events. Relatedly, it is possible that the calendar date condition was more effective at spontaneously evoking EFT than the calendar unit condition. That is, participants may be more likely to discriminate future events per the date of the event (e.g., "I start my new job on September 15, 2020.") compared to the units of time until the event (e.g., "I start my new job in 6 months."). This explanation is speculative 
and is subject to empirical investigation; however, the results of the current study indicate that durations framed as dates decrease discounting in a similar manner to EFT. Future research should investigate the extent to which duration framing and EFT rely on similar behavioral mechanisms.

Second, it is possible that participants perceived compliance with the hypothetical social-distancing policies as more feasible in the calendar date condition compared to the calendar unit condition. That is, when a delay or duration is framed in units typically associated with waiting (e.g., you need to wait 2 weeks until your next paycheck), the delay may be perceived as longer compared to when the delay is framed as a date, which is typically associated with the delivery of reinforcement (e.g., you get paid on March 31). That is, when presented with an option for delayed reinforcement, participants may be more likely to attend to the delayed component when calendar units are used and more likely to attend to the reinforcement component when calendar dates are used. The differences in attention may subsequently evoke verbal behavior associated with either (a) the response cost of waiting (P. Johnson, Hermann, \& Johnson, 2015) or (b) the reward value of waiting, respectively. Thus, the calendar unit condition may have yielded higher rates of compliance to hypothetical social-distancing policies because participants attended to the reward component (i.e., reducing rates of infection and staying healthy) rather than the delayed component (i.e., response cost or effort of abstaining from sources of immediate social reinforcement). Future research may provide preliminary evidence for this explanation by instructing participants to "think aloud" (Hayes et al., 1998) while responding to delay-discounting tasks. Participants' verbal behavior could then be coded to determine if attending is primarily delay based (e.g., "I would have to wait 6 months to get [reward].") or reward based (e.g., "I would get [reward] on the same date that I start my new job.").

Finally, it is possible participants perceived delayed rewards framed as calendar dates as more certain than rewards framed as calendar units. That is, calendar dates may have promoted the perception that the hypothetical social-distancing policy would definitely end on a specific date. In contrast, calendar units may have promoted the perception that the hypothetical social-distancing policy may end after a specific duration. Participants' local reinforcement history with actual social-distancing policies may have further promoted this perception. That is, between March 15, 2020, and the conclusion of the study (May 15, 2020), many states enacted several "rounds" of 2week social-distancing policies. For example, at the conclusion of the first 2 weeks of social distancing, a second 2 weeks was enacted, and then a third, and so on. It is possible that this local pairing with unit-based durations and uncertainty contributed to the decreased rates of compliance in the calendar unit condition. In the context of delay discounting, levels of uncertainty decrease the subjective value of the delayed outcome (Cox \& Dallery, 2016). For example, Cox and Dallery (2016) used a repeated-measures design to assess the extent to which systematic changes in the certainty of a delayed reward (i.e., $10 \%$ certainty to $100 \%$ certainty) affected the rate at which participants discounted the value of a delayed hypothetical monetary reward. As more uncertainty was introduced to the delivery of the delayed reward, participants assigned relatively decreased subjective value to the delayed reward, which resulted in steeper discounting curves. Thus, it is possible that participants assigned lower subjective value to complying in the calendar unit condition because of the perceived uncertainty in the termination of the hypothetical social-distancing policy. This interpretation is subject 
to empirical validation; however, the local reinforcement histories of many of the participants and the steeper discounting curve in the calendar unit condition provide preliminary and anecdotal support for this interpretation.

The general goal of the current study was to demonstrate the utility of a discounting framework in analyzing variables that affect compliance with social-distancing policies. As such, the results indicate that the differential framing of durations of hypothetical social-distancing policies exerts similar effects on self-reported compliance as it does in monetary-discounting scenarios (e.g., DeHart \& Odum, 2015). However, the current study contains several limitations that need to be addressed. First, contextual factors at the time of the experiment may have controlled participants' responding. That is, the experiment was conducted during the COVID-19 pandemic while many individuals were currently contacting and practicing social-distancing policies. Thus, it is possible that the current social context may have served as a motivating operation for compliance with hypothetical social-distancing policies. Similarly, Ostaszewski, Green, and Myerson (1998) found that hypothetical monetary-discounting rates were affected by actual economic fluctuations in inflation in Poland (circa 1994). Participants completed discounting questionnaires using American currency (i.e., U.S. dollar) and Polish currency (i.e., zloty) at the height of economic inflation in Poland. Participants' rates of discounting were far steeper for zlotys compared to U.S. dollars, indicating that contextual factors - in this case, economic contextual factors - altered the effect of the programmed independent variable (i.e., delay to the reward). Similarly, it is possible that societal contextual factors altered the effect of the current study's programmed independent variables (i.e., frame and duration of the policy).

Relatedly, it is likely that some proportion of the participants had previously demonstrated compliance with actual social-distancing policies at the durations used in the current study. For example, participants may have completed the questionnaire while concurrently complying with state-mandated social-distancing policies. Thus, participants' actual levels of compliance with actual concurrent social-distancing policies may have controlled responses to hypothetical social-distancing policies. It is unclear, however, if this limitation enhances the external validity (similar to M. W. Johnson \& Bickel, 2002) or diminishes the external validity (similar to Dixon, Mui Ker Lik, Green, \& Myerson, 2013) of these findings. Future research should attempt to replicate the current study under different contexts and time frames to determine the effect of local reinforcement histories.

Second, the current study's dependent variable, self-reported percentage of compliance, contains ambiguity that may limit interpretations. That is, values of compliance do not necessarily correlate to specific components of the hypothetical social-distancing policies. For self-reported levels of compliance between $1 \%$ and $99 \%$, it is unclear which components participants would demonstrate compliance with and which components participants would not demonstrate compliance with. This is similar to the study by Plumm et al. (2012), in which participants reported percentages of a "perfect" hypothetical policy to be implemented immediately; it is unclear which components of the policy participants were willing to compromise for immediate implementation. Similar limitations are present in treatment integrity research that omit descriptive assessments. Findings from studies that systematically manipulate components of treatment integrity generally conclude that reduced integrity with different components correlates to different response outcomes (see Brand, Henley, DiGennaro Reed, Gray, 
\& Crabbs, 2019, for a review). Decrements in treatment integrity for some components exert large effects, whereas decrements in treatment integrity for other components exert small effects. Similarly, compliance with different components of socialdistancing policies may exert different effects on the intervention's effectiveness. For example, full compliance with social isolation (i.e., not leaving one's home) may nullify noncompliance with wearing a face mask in public. Though the current study provided general information on the components involved in social-distancing policies, it did not provide a comprehensive task analysis or opportunities for participants to report compliance with different components. Future research should measure hypothetical levels of compliance with different components of social policies.

Third, the current study used $80 \%$ compliance as the cutoff between high integrity (i.e., compliance with the hypothetical policy) and low integrity (i.e., noncompliance with the hypothetical policy). Though $80 \%$ is a common measure of treatment integrity in skill acquisition interventions (Bottini et al., 2020), it is somewhat arbitrary in the context of compliance with social policies. Similar to parametric evaluations of treatment integrity (e.g., Hirst \& DiGennaro Reed, 2015), future research should investigate methods to measure the effects of different rates of compliance with social policies to determine efficacious levels of treatment integrity.

The current study evaluated the effects of time framing on rates of compliance within a discounting framework. This study applied a discounting framework to explain changes in compliance across increasing durations of hypothetical policies. That is, participants discounted the subjective value of sustained compliance across increasing durations of social distancing. These results, along with the work by Plumm et al. (2012), enhance the utility and generalizability of discounting frameworks to describe diverse behavioral phenomena. Finally, the current study proposed an understanding of compliance with hypothetical social-distancing policies as a measure of treatment integrity. This conceptualization may prove productive, as it concerns identifying variables that enhance or diminish measures of treatment integrity.

In the context of this study, hypothetical social-distancing policies with durations framed as calendar dates yielded an average of $10 \%$ more compliance than durations framed as calendar units. Extrapolated to the scale of the population of the United States (approximately 330,000,000, circa 2020; U.S. Census Bureau, 2020), framing a social-distancing policy using calendar dates may result in 33,000,000 more citizens complying with the policy.

\section{Compliance with Ethical Standards}

Conflict of interest The author reports no conflict of interest.

Ethical approval This research was approved by Briar Cliff University’s Institutional Review Board (00302020).

\section{Calendar Unit Questionnaire}

DELAYS: 1 DAY, 1 WEEK, 2 WEEKS, 1 MONTH, 3 MONTHS, 6 MONTHS, 1 YEAR 
A team of internationally renowned medical doctors state that the severity of the COVID-19 pandemic will be significantly reduced to manageable levels (i.e., similar to the management of the seasonal influenza virus) if citizens completely socially distance (i.e., confine themselves to contact just with individuals in their household) for [DELAY]. How likely are you to comply with this social-distancing policy?

[Participant adjusts slider ranging from $0 \%$ to $100 \%$.]

\section{Calendar Date Questionnaire}

DELAYS: MARCH 16, 2020, MARCH 22, 2020, MARCH 29, 2020, APRIL 15, 2020, JUNE 15, 2020, SEPTEMBER 15, 2020, DECEMBER 15, 2020, MARCH 15, 2021

A team of internationally renowned medical doctors state that the severity of the COVID-19 pandemic will be significantly reduced to manageable levels (i.e., similar to the management of the seasonal influenza virus) if citizens completely socially distance (i.e., confine themselves to contact just with individuals in their household) until [DELAY]. How likely are you to comply with this social-distancing policy?

[Participant adjusts slider ranging from $0 \%$ to $100 \%$.]

\section{References}

Atance, C., \& O’Neil, D. K. (2001). Episodic future thinking. Trends in Cognitive Sciences, 5, 533-539. https://doi.org/10.1016/s1364-6613(00)01804-0.

Behrend, T. S., Sharek, D. J., Meade, A. W., \& Wiebe, E. N. (2011). The viability of crowdsourcing for survey research. Behavioral Research Methods, 43(8), 800-813. https://doi.org/10.3758/s13428-0110081-0.

Bickel, W. K., Jarmolowicz, D. P., Mueller, E. T., Koffarnus, M. N., \& Gatchalian, K. M. (2012). Excessive discounting of delayed reinforcers as a trans-disease process contributing to addiction and other diseaserelated vulnerabilities: Emerging evidence. Pharmacology \& Therapeutics, 134(3), 287-297. https://doi. org/10.1016/j.pharmthera.2012.02.004.

Bickel, W. K., \& Marsch, L. (2001). Toward a behavioral economic understanding of drug dependence: Delay discounting processes. Addiction, 96, 32-47.

Bottini, S., Morton, H., Gillis, J., \& Romanczyk, R. (2020). The use of mixed modeling to evaluate the impact of treatment integrity on learning. Behavioral Interventions, 35, 372-391. https://doi.org/10.1002/bin. 1718 .

Brand, D., Henley, A. J., DiGennaro Reed, F. D., Gray, E., \& Crabbs, B. (2019). A review of published studies involving parametric manipulations of treatment integrity. Journal of Behavioral Education, 28, 1-26. https://doi.org/10.1007/s10864-018-09311-8.

Bromberg, U., Lobatcheva, M., \& Peters, J. (2017). Episodic future thinking reduces temporal discounting in healthy adolescents. PLOS One, 12(1), 1-15. https://doi.org/10.1371/journal.pone.0188079.

Centers for Disease Control and Prevention. (2020). Social distancing [News release]. https://www.cdc.gov/ coronavirus/2019-ncov/prevent-getting-sick/social-distancing.html

Cohen, J. (1988). Statistical power analysis for the behavioral sciences. Routledge Academic.

Collado, A., Johnson, P. S., Loya, J. M., Johnson, M. W., \& Yi, R. (2017). Discounting of condom-protected sex as a measure of high risk for sexually transmitted infection among college students. Archives of Sexual Behavior, 46(7), 2187-2195. https://doi.org/10.1007/s10508-016-0836-x.

Cox, D. J., \& Dallery, J. (2016). Effects of delay and probability combinations on discounting in humans. Behavioural Processes, 131, 15-23. https://doi.org/10.1016/j.beproc.2016.08.002.

DeHart, W. B., \& Odum, A. L. (2015). The effects of the framing of time on delay discounting. Journal of the Experimental Analysis of Behavior, 103, 10-21. 
Diaz, R. M., \& Berk, L. E. (1992). Private speech: From social interaction to self-regulation. Lawrence Erlbaum Associates.

Dixon, M. R., Mui Ker Lik, N., Green, L., \& Myerson, J. (2013). Delay discounting of hypothetical and real money: The effect of holding reinforcement rate constant. Journal of Applied Behavior Analysis, 46(2), 512-517. https://doi.org/10.1002/jaba.42.

Epstein, L. H., Salvy, S. J., Carr, K. A., Dearing, K. K., \& Bickel, W. K. (2010). Food reinforcement, delay discounting and obesity. Physiology \& Behavior, 100, 438-445.

Hardisty, D. J., Johnson, E. J., \& Weber, E. U. (2010). A dirty word or a dirty world? Attribute framing, political affiliation, and query theory. Psychological Science, 21, 86-92. https://doi.org/10.1177/ 0956797609355572.

Hardisty, D. J., \& Weber, E. U. (2009). Discounting future green: Money versus the environment. Journal of Experimental Psychology: General, 138, 329-340. https://doi.org/10.1037/a0016433.

Hayes, S. C., White, D., \& Bissett, R. T. (1998). Protocol analysis and the "silent dog" method of analyzing the impact of self-generated rules. The Analysis of Verbal Behavior, 15, 57-63.

Hirst, J. M., \& DiGennaro Reed, F. D. (2015). An examination of the effects of feedback accuracy on academic task acquisition in analogue settings. The Psychological Record, 65, 49-65. https://doi.org/10. 1007/s40732-014-0087-y.

Jackson, J. N. S., \& MacKillop, J. (2016). Attention-deficit/hyperactivity disorder and monetary delay discounting: A meta-analysis of case-control studies. Biological Psychiatry: Cognitive Neuroscience and Neuroimaging, 1(4), 316-325. https://doi.org/10.1016/j.bpsc.2016.01.007.

Johnson, M. W., \& Bickel, W. K. (2002). Within-subject comparison of real and hypothetical money rewards in delay discounting. Journal of the Experimental Analysis of Behavior, 77, 129-146. https://doi.org/10. 1901/jeab.2002.77-129.

Johnson, P., Hermann, E., \& Johnson, M. (2015). Opportunity costs of reward delays and the discounting of hypothetical money and cigarettes. Journal of the Experimental Analysis of Behavior, 103, 87-107.

Kirby, K. N., \& Marakovic, N. N. (1996). Delay-discounting probabilistic rewards: Rates decrease as amounts increase. Psychonomic Bulletin \& Review, 3, 100-104. https://doi.org/10.3758/BF03210748.

Kirby, K. N., Petry, N. M., \& Bickel, W. K. (1999). Heroin addicts have higher discount rates for delayed rewards than non-drug-using control. Journal of Experimental Psychology: General, 128, 78-87.

Koffarnus, M. N., Jarmolowicz, D. P., Mueller, E. T., \& Bickel, W. K. (2013). Changing delay discounting in the light of the competing neurobehavioral decision systems theory: A review. Journal of the Experimental Analysis of Behavior, 99, 32-57.

LeBoeuf, R. A. (2006). Discount rates for time versus dates: The sensitivity of discounting to time-interval description. Journal of Marketing Research, 43, 59-72. https://doi.org/10.1509/jmkr.43.1.59.

Madden, G. J., \& Johnson, P. S. (2010). A delay discounting primer. In G. J. Madden \& P. S. Johnson (Eds.), Impulsivity: The behavioral and neurological science of discounting (pp. 11-37). American Psychological Association.

Mazur, J. E. (1985). Probabilistic and delay of reinforcement as factors in discrete-trial choice. Journal of the Experimental Analysis of Behavior, 43(3), 341-351.

McKerchar, T. L., \& Renda, C. R. (2012). Delay and probability discounting in humans: An overview. The Psychological Record, 62, 817-834. https://doi.org/10.1007/BF03395837.

Mishra, S., \& Lalumière, M. L. (2017). Associations between delay discounting and risk-related behaviors, traits, attitudes, and outcomes. Journal of Behavioral Decision Making, 30, 769-781.

Myerson, J., Green, L., \& Warusawitharana, M. (2001). Area under curve as a measure of delay discounting. Journal of the Experimental Analysis of Behavior, 76, 235-243. https://doi.org/10.1901/jeab.2001.76235.

Ostaszewski, P., Green, L., \& Myerson, J. (1998). Effects of inflation on the subjective value of delayed and probabilistic rewards. Psychonomic Bulletin \& Review, 5(2), 324-333.

O’Donnell, S., Hollis-Hansen, K., \& Epstein, L. (2019). Mix and match: An investigation into whether episodic future thinking cues need to match discounting delays in order to be effective. Behavioral Sciences, 9(1), 1-9. https://doi.org/10.3390/bs9010001.

Petry, N. M., \& Madden, G. J. (2010). Discounting and pathological gambling. In G. J. Madden \& W. K. Bickel (Eds.), Impulsivity: The behavioral and neurological science of discounting (pp. 273-294). American Psychological Association.

Plumm, K. M., Borhart, H., \& Weatherly, J. N. (2012). Choose your words wisely: Delay discounting of differently titled social policy issues. Behavior and Social Issues, 21, 26-48.

Rattner, A., Yagil, D., \& Shermn-Segal, C. (2003). The sense of entitlement to violate the law: Legal disobedience as a public versus private reaction. Social Behavior and Personality, 31, 545-556. https:// doi.org/10.2224/SBP.2003.31.6.545. 
Read, D., Frederick, S., Orsel, B., \& Rahman, J. (2005). Four score and seven years from now: The date/delay effect in temporal discounting. Management Science, 51(9), 1326-1335. https://doi.org/10.1287/mnsc. 1050.0412 .

Rung, J. M., \& Madden, G. J. (2018). Experimental reductions of delay discounting and impulsive choice: A systematic review and meta-analysis. Journal of Experimental Psychology: General, 147(9), 1349-1381. https://doi.org/10.1037/xge0000462.

St. Peter Pipkin, C., Vollmer, T. R., \& Sloman, K. N. (2010). Effects of treatment integrity failures during differential reinforcement of alternative behavior: A translational model. Journal of Applied Behavior Analysis, 43, 47-70. https://doi.org/10.1901/jaba.2010.43-47.

U.S. Census Bureau. (2020). U.S. and world population clock. https://www.census.gov/popclock/

Weatherly, J. N. (2010). Delay discounting of different commodities varies as a function of political party affiliation in a college sample. Behavior and Social Issues, 19, 167-178. https://doi.org/10.5210/bsi. v19i0.3080.

World Health Organization. (2020). Coronavirus disease (COVID-19) advice for public [News release]. https://www.who.int/emergencies/diseases/novel-coronavirus-2019/advice-for-public 